especially in younger age groups. This information would allow us to better allocate resources (e.g. provide an SMS messaging platform) and tailor PN methods according to age groups, sexual preference.

Disclosure of interest statement The authors report no conflict of interest in this study. The department of STI Control Clinic is funded by Ministry of Health, Singapore. No pharmaceutical grants were received in the development of this study.

\section{P04.04 CHLAMYDIA INFECTION IN MALES AND FEMALES REPORTING SEX WITH PARTNERS WITH CHLAMYDIA}

1,2,3,4S Huffam*, ${ }^{1,5}$ EPF Chow, ${ }^{1,5} \mathrm{CK}$ Fairley, ${ }^{2}$ J Hocking, ${ }^{1} \mathrm{~J}$ Peel, ${ }^{1,5} \mathrm{M}$ Chen. ${ }^{1}$ Melbourne Sexual Health Centre, Alfred Health, Melbourne, Victoria, Australia; ${ }^{2}$ School of Population and Global Health, University of Melbourne, Victoria, Australia; ${ }^{3}$ Department of Infectious Diseases, Barwon Health, Victoria, Australia; ${ }^{4}$ Royal Darwin Hospital, Northern Territory, Australia; ${ }^{5}$ Central Clinical School, Faculty of Medicine, Nursing and Health Sciences, Monash University, Victoria, Australia

\subsection{6/sextrans-2015-052270.258}

Background Justification for presumptive treatment for a sexually transmitted infection depends in part on the underlying prevalence of that infection among those reporting exposure. To examine these data for chlamydia we aimed to ascertain the proportion infected with chlamydia, and factors predictive of infection, amongst females, heterosexual males and men who have sex with men (MSM) presenting to a sexual health service reporting sexual contact with a chlamydia infected partner.

Methods Patients included were those attending the Melbourne Sexual Health Centre from October 2010 to September 2013. Proportions testing positive amongst females, heterosexual males, and MSM reporting sexual contact with a chlamydia infected partner were ascertained. Demographic and behavioural data obtained using computer assisted self-interview were analysed to determine predictive factors.

Results Of the 491 female, 808 heterosexual male, and 268 MSM chlamydia contacts, the proportion diagnosed with chlamydia were $39.9 \%$ (95\% CI 35.7-44.3), 36.1\% (95\% CI 32.939.9 ), and $23.5 \%$ (95\% CI 18.8-29.0) respectively. Female chlamydia contacts were more likely to have chlamydia if age $\leq 24$ (AOR 1.86, 95\% CI 1.12-3.10) or if they reported inconsistent condom use during vaginal sex with a regular male partner (AOR 2.5, 95\% CI 1.12-6.14). Heterosexual male contacts were more likely to have chlamydia if age $\leq 26$ (AOR $1.70,95 \%$ CI 1.26-2.30) or if they had a regular female sexual partner (AOR $1.42,95 \%$ CI 1.06-1.91). In MSM urethral chlamydia was diagnosed in $8.8 \%$, rectal chlamydia in $20.2 \%$, and in $3.9 \%$ at both sites. MSM were more likely to have chlamydia if they had a regular male sexual partner (OR 2.12 95\% CI 1.18-3.81).

Conclusion This study of female, heterosexual male and MSM presentations with self-reported chlamydia contact provides insight as to their likelihood of infection. The data may inform policy and individual clinical decision making regarding presumptive treatment of chlamydia contacts.

Disclosures No potential conflicts of interest.

\section{P04.05 HIGH PREVALENCE OF RECTAL GONORRHOEA AMONG MEN REPORTING CONTACT WITH MEN WITH GONORRHOEA: IMPLICATIONS FOR EPIDEMIOLOGICAL TREATMENT}

${ }^{1} \mathrm{~K}$ Dutt, ${ }^{1,2}$ EPF Chow*, ${ }^{*} \mathrm{~S}$ Huffam, ${ }^{1,3} \mathrm{~K}$ Klassen, ${ }^{1,2} \mathrm{CK}$ Fairley, ${ }^{1,2} \mathrm{CS}$ Bradshaw, ${ }^{1} \mathrm{I}$ Denham, ${ }^{1}$ MY Chen*. 'Melbourne Sexual Health Centre, Alfred Hospital, Melbourne, Victoria, Australia; ${ }^{2}$ Central Clinical School, Monash University, Melbourne, Victoria, Australia;

${ }^{3}$ Faculty of Medicine, Melbourne University, Melbourne, Victoria, Australia

10.1136/sextrans-2015-052270.259

Introduction This study aimed to determine the prevalence of gonorrhoea and factors associated with rectal gonorrhoea among men reporting sexual contact with men with gonorrhoea.

Methods Men who presented to Melbourne Sexual Health Centre reporting sexual contact with a male with gonorrhoea were prospectively identified between March 2011 and December 2013. These men were screened for pharyngeal and rectal gonorrhoea using culture. The prevalence of gonorrhoea among contacts was compared to that among all men who have sex with men (MSM) screened at the clinic over the same period. Logistic regression was performed on demographic and behavioural characteristics to determine the predictors of infection among contacts.

Results Among 363 contacts of gonorrhoea the prevalence of rectal gonorrhoea was 26.4\% (95\% CI: 21.8\%-31.0\%) compared to $3.9 \%$ (95\% CI: 3.7\%-4.2\%) among clinic attendees (p $<0.001$ ). The prevalence of pharyngeal gonorrhoea among contacts was 9.4\% (95\% CI: 6.4\%-12.4\%) compared to $2.1 \%$ (95\% CI: 1.9\%-2.4\%) among clinic attendees ( $p<0.001)$. Among contacts who reported not always using condoms during receptive anal sex with casual partners, rectal gonorrhoea was cultured in $42.4 \%$ compared with $12.7 \%$ among contacts reporting no receptive anal sex $(\mathrm{p}<0.001)$ and $20.2 \%$ among those reporting always using condoms $(\mathrm{p}<0.001)$. On multivariate analysis rectal gonorrhoea was associated with inconsistent condom use during receptive anal sex with casual partners (adjusted odds ratio (AOR): 4.16; 95\% CI: 1.87-9.26) and a reported past history of gonorrhoea (AOR: 1.77; 95\% CI: 1.01-3.14).

Conclusion The high proportion of positive cases of gonorrhoea among contacts in this study supports epidemiological treatment of MSM presenting as contacts of gonorrhoea.

Disclosure of interest There are no conflicts of interest to be disclosed relating to this paper.

\section{P04.06 PARTNER NUMBER AND OUTCOMES OF PARTNER NOTIFICATION AMONG GAY, BISEXUAL, AND OTHER MEN WHO HAVE SEX WITH MEN WITH INFECTIOUS SYPHILIS IN BRITISH COLUMBIA, CANADA}

${ }^{1,2} \mathrm{~J}$ Wong ${ }^{*},{ }^{1} \mathrm{M}$ Achen, ${ }^{1} \mathrm{~N}$ Holgerson, ${ }^{1,2} \mathrm{G}$ Ogilvie, ${ }^{1,2,3} \mathrm{M}$ Gilbert. ${ }^{1} B C$ Centre for Disease Control; ${ }^{2}$ University of British Columbia; ${ }^{3}$ Ontario HIV Treatment Network

\subsection{6/sextrans-2015-052270.260}

Background Since 2010, infectious syphilis (IS) cases in British Columbia (BC) have increased 4-fold among gay, bisexual, and 